\title{
ASOCIACIONES DE INMIGRANTES EN LA ENCRUCIJADA. ACCIÓN TRANSNACIONAL Y RIESGOS DE COOPTACIÓN
}

\author{
Associations among immigrants at the crossroads. \\ Transnational action and co-option risks
}

Joan Lacomba ${ }^{1}$

\begin{abstract}
Resumen. En este artículo tratamos de contribuir al debate en torno al papel de las asociaciones de inmigrantes en las sociedades de origen y destino, así como puente entre las mismas. El creciente número y potencial de las asociaciones ha puesto de relieve su contribución al desarrollo y a la dinamización de las sociedades civiles de uno y otro lado. Sin embargo, este proceso no está exento de limitaciones y dinámicas que pueden entrar en contradicción con el papel fundacional de las mismas asociaciones. La interacción con las instituciones de los países de origen de origen y destino, y el acceso a programas y fondos públicos estatales, habría generado un escenario en el que las asociaciones han experimentado cambios organizativos y en su relación con el poder que han sido objeto de crítica.
\end{abstract}

Palabras clave: asociaciones, inmigrantes, Estado, desarrollo e integración.

\begin{abstract}
This paper contributes to the debate on the role of the associations among immigrants in their origin and destination countries, working as a bridge between them. The growing number and the potential of these associations have highlighted their contribution to the development and the renewal of the civil societies of the origin and destination countries. However, this process still has limitations and dynamics that may conflict with the basic role of these associations. The interaction between the origin and destination countries, as well as the access to governmental programs and funds, has created a scenario in which these associations have undergone organizational and power-related changes, which have been an object of criticism.
\end{abstract}

Keywords: associations, immigrants, State, development and integration.

Universidad de Valencia. Valencia, España. 


\section{Introducción ${ }^{2}$}

Bajo el influjo de los debates sobre el papel de la sociedad civil y sus relaciones con el Estado, las asociaciones de diverso tipo se han constituido en objeto de estudio frecuente. En menor medida, pero de forma creciente, las asociaciones de inmigrantes también han despertado el interés de los investigadores sociales, en especial en el contexto de los países de recepción de la población inmigrante, así como por parte de los estudiosos locales de los propios países de origen. Un buen número de los trabajos sobre las asociaciones constituidas por personas inmigrantes se ha centrado en el papel de las mismas en los contextos de recepción, fundamentalmente en cuanto a su función integradora. En este artículo nos centramos en el papel que las asociaciones de inmigrantes pueden jugar tanto en relación con sus sociedades de destino como de origen, a través de su participación e integración en la vida social y política y, eventualmente, su capacidad de agencia a la hora de condicionar políticas y dinámicas locales.

La popularización del enfoque de capacidades y el concepto de agencia en numerosos ámbitos, ha acompañado a cambios políticos y en las estructuras institucionales que han sido presentados precisamente como una forma de aumentar también la capacidad de agencia de los inmigrantes. Esta cuestión no puede desligarse de las directrices que los organismos internacionales de desarrollo han extendido en torno al empoderamiento y fortalecimiento de grupos excluidos, entre ellos las mismas poblaciones inmigrantes. Tanto organismos internacionales, como instituciones nacionales de los Estados de destino y de origen, se han manifestado en esta línea o incluso han promovido medidas y programas dirigidos a incrementar el papel y la agencia de las asociaciones de inmigrantes en el desarrollo. Sin embargo, la agencia entendida como libertad para actuar ${ }^{3}$ choca con la pretensión de "empoderar" a las asociaciones en una dirección y con un objetivo determinado, a menudo no compartido por las mismas. Todo este proceso de reajuste político en torno a las asociaciones no puede explicarse al margen del reposicionamiento de los Estados de destino y de origen de los inmigrantes y de sus propias sociedades civiles, en un contexto de revisión de sus papeles y en un marco de crecientes interconexiones entre unos y otros escenarios pese a las distancias geográficas.

Tanto en los lugares de destino como de origen las denuncias de relaciones clientelares entre gobiernos y asociaciones de inmigrantes han sido frecuentes,

2 Este artículo recoge resultados del proyecto de investigación $(I+D+I)$ "Diásporas y codesarrollo desde España. El papel de las asociaciones de inmigrantes en el desarrollo de sus países de origen", financiado por el Ministerio de Ciencia e Innovación, CSO2011-22686. También incorpora reflexiones generadas dentro del proyecto de investigación en curso "El aporte del asociacionismo migrante a la sociedad civil española. Nuevos modelos organizativos y de relación con las Organizaciones No Gubernamentales de Desarrollo", CSO2015-66181-R (MINECO/FEDER).

SEN, Amartya. Desarrollo y libertad. 
aunque quizás no más excepcionales que las que han afectado a otros tipos de Organizaciones No Gubernamentales. De hecho, el cuestionamiento de la labor sustitutiva del Estado ejercida por las asociaciones de inmigrantes se habría hecho extensivo a partir de la crítica muchos más profunda al papel de las Organizaciones No Gubernamentales de Desarrollo en países en desarrollo ${ }^{4}$. En España, el trabajo de González y Morales ${ }^{5}$ sobre las asociaciones de inmigrantes en Madrid, o las investigaciones de Veredas ${ }^{6}$ y Toral $^{7}$ sobre la integración de las asociaciones de inmigrantes en plataformas de participación impulsadas desde las instancias oficiales, han puesto de relieve las prácticas clientelares y los riesgos de cooptación política de las mismas asociaciones. Del mismo modo, en importantes países de origen de los migrantes como México o Marruecos se han detectado problemas similares ${ }^{8}$.

Para ilustrar estas cuestiones, y discutir algunos de los supuestos comúnmente aceptados, tomamos el caso de las asociaciones de una serie de países de características distintas con flujos significativos de inmigrantes hacia España, y que nos sirven para mostrar, desde una óptica transnacional, los retos a los que se enfrentan a partir de una diversidad de situaciones, lógicas y resultados. El estudio que sirve de base para estas páginas se desarrolló entre los años 2013 y 2015 entre las asociaciones de inmigrantes de Colombia, Ecuador, Marruecos y Senegal presentes en España, a través de una amplia encuesta prospectiva y la posterior realización de entrevistas a las asociaciones implicadas en acciones en sus países de origen. En esta ocasión, más allá de los datos presentados en otros trabajos, recogemos aquí algunos de los debates teóricos y reflexiones que acompañaron a nuestra propia investigación y que, como es lógico, fueron alimentados por nuestros propios hallazgos, pero también se nutrieron de las investigaciones preexistentes a las que nos referiremos a continuación.

\section{Asociaciones de inmigrantes y sociedades de destino}

La propensión de los inmigrantes a agruparse y organizarse de distintos modos en los países de asentamiento forma parte de la historia contemporánea de los flujos migratorios. En todos los países de recepción los inmigrantes han tendido a crear estructuras organizativas que diesen respuesta a sus necesidades en formas muy variadas, como sociedades secretas, asociaciones

\footnotetext{
BRETÓN, Víctor. Cooperación al desarrollo y demandas étnicas en los andes ecuatorianos.

GONZÁLEZ, Amparo, MORALES, Laura. Las asociaciones de inmigrantes en Madrid. Una nota de investigación sobre su grado de integración política.

6 VEREDAS, Sonia. Las asociaciones de inmigrantes en España. Práctica clientelar y cooptación política.

7 TORAL, Guillermo. Las asociaciones de inmigrantes como sociedad civil: un análisis tridimensional.

8 GOLDRING, Luin. The mexican state and transmigrant organizations: Negotiating the boundaries of membership and participation; ISKANDER, Natasha. Creative State: Forty Years of Migration and Development Policy in Morocco and Mexico.
} 
en torno a créditos rotatorios, sociedades de ayuda mutua, asociaciones religiosas, asociaciones de paisanos o grupos políticos, entre otras ${ }^{9}$. Tal como han mostrado los diferentes estudios, el asociacionismo forma parte del proceso de incorporación de los migrantes como minoría étnicas en las sociedades de acogidas y no contradice necesariamente su voluntad de integración. Como Castles y Miller nos recuerdan, "los grupos étnicos surgidos de la inmigración necesitan sus propias asociaciones y redes sociales, al igual que sus propios idiomas y culturas. Las políticas que niegan legitimidad a estas necesidades llevan al aislamiento y el separatismo"10.

Los primeros trabajos sistemáticos llevados a cabo sobre las formas modernas de las asociaciones de inmigrantes, tanto en Europa ${ }^{11}$, como en los Estados Unidos ${ }^{12}$, han puesto de relieve que estas juegan un papel adaptativo fundamental para los recién llegados, aunque sus formas de articulación con la sociedad y las instituciones de acogida puedan variar en función de las variables que operan dentro de cada colectivo (origen rural-urbano, perfil cualificado-no cualificado, posición de clase o religión). Sin embargo, y aunque la mayor parte de la literatura sobre asociaciones de inmigrantes haya destacado la función integradora de las mismas en la sociedad de acogida y realizado una valoración positiva de ello, algunos trabajos también han mostrado los riesgos asociados a sus interacciones en el ámbito más político.

En este sentido, el papel de las asociaciones de inmigrantes ha sido frecuentemente, cuanto menos, contradictorio, y el coste de su reconocimiento como agentes sociales y políticos en la sociedad de acogida ha sido muchas veces el de la creación de formas de clientelismo y la cooptación de sus líderes. Castles y Miller explican cómo "las agencias gubernamentales en ocasiones veían a las organizaciones étnicas tradicionalistas como instrumentos efectivos para el control social de los trabajadores, los jóvenes o las mujeres", y también cómo "los líderes étnicos podían usar su nuevo papel para preservar la autoridad tradicional y hacer más lento el cambio tanto cultural como político". De este modo, dicen, "el Estado podía escoger con cuáles líderes étnicos trabajaría y cuáles ignoraría, y recompensar el comportamiento deseado a través de su patrocinio y financiamiento". Obviamente, la cooptación es un proceso doble: "las agencias del Estado intentaban utilizar estructuras y asociaciones de las comunidades étnicas, si bien, a cambio, debían hacer concesiones"13. Del mismo modo, el trabajo pionero de Rex indica que, tras un primer período en que las asociaciones

\footnotetext{
9 MOYA, José C. Immigrants and associations: a global and historical perspective.

${ }^{10}$ CASTLES, Stephen, MILLER, Mark J. La era de la migración Movimientos internacionales de población en el mundo moderno, p. 306.

${ }^{11}$ REX, John, JOLY, Danielle, WILPERT, Czarina. Immigrant Associations in Europe.

12 SASSEN-KOOB, Saskia. Formal and informal associations: Dominicans and Colombians in New York.

${ }^{13}$ CASTLES, MILLER, op. cit., p. 364.
} 
proveen las necesidades de los inmigrantes, éstas pasan a buscar los beneficios del Estado para cumplir con esa función, lo que se acompañaría de nuevas formas de clientelismo y el patronazgo ${ }^{14}$. Por su parte, Moya argumenta que la pregunta sobre si las intervenciones gubernamentales desaniman o promueven la actividad de las asociaciones de inmigrantes no puede ser respondida en términos afirmativos o negativos, sino que depende de factores históricos y situacionales. Para Moya millones de inmigrantes de todo el mundo han desarrollado estructuras organizativas impresionantes con poco o ningún apoyo del Estado que, afirma, "no sea la concesión de la libertad asociativa"15. Es más, sostiene que el apoyo logístico y financiero gubernamental pueden promover la formación de determinados tipos de organizaciones que proporcionan servicios y defienden los derechos de grupos, así como ayudar a su incorporación política, pero la participación del gobierno tiene poco o ningún efecto sobre la formación de grupos recreativos y sociales, que siguen representando, dice, la forma más común de las asociaciones voluntarias en las diásporas ${ }^{16}$.

Sea como sea, muchas de las asociaciones de inmigrantes viven en una tensión permanente entre su actuación como entes prestadores de servicios diversos - algunas pueden acabar equiparadas a empresas de servicios- y su faceta más reivindicativa frente a las administraciones y la defensa de derechos de los propios migrantes ${ }^{17}$. El desempeño de su función más instrumental (la primera) puede llevar a disponer de una amplia base de asociadosbeneficiarios, al tiempo que un reconocimiento social e, incluso, ser aceptados como interlocutores políticos por parte de las autoridades; en cambio, su labor más reivindicativa puede generar fracturas en la representación de los mismos migrantes (no todos se posicionan del mismo modo) y generar conflictos con el poder político, tendente a alinearse con las asociaciones afines a sus planteamientos. El coste del primer escenario suele ser la dependencia o la sumisión con el fin de lograr mayores recursos, pero el coste en el segundo caso puede ser mucho más elevado: el aislamiento y la exclusión con el riesgo asociado de desaparición. Se trataría de la disyuntiva clásica establecida por Hirschman ${ }^{18}$ en torno a los tres posibles escenarios que se plantean a las organizaciones en relación con los vínculos con el poder: el "exit, voice and loyaltie", o las posibilidades de permanecer al margen, integrarse con voz propia y alinearse con los dictados institucionales.

\footnotetext{
14 REX, op. cit., p. 10.

${ }^{15}$ MOYA, op. cit., p. 856.

16 Ibidem, p. 857.

17 LUSSI, Carmem, MARINUCCI, Roberto. O protagonismo dos brasileiros no exterior e seu papel na defesa e promoção dos próprios direitos. Resultados de uma pesquisa junto a organizações e lideranças de brasileiros e brasileiras no exterior, p. 140.

${ }^{18}$ HIRSCHMAN, Albert O. Salida, voz y lealtad. Respuestas al deterioro de empresas, organizaciones y estados.
} 
Esta cuestión ha sido discutida ampliamente en el contexto español, donde las asociaciones de inmigrantes han ocupado un destacado lugar en las investigaciones académicas ${ }^{19}$ y en las mismas políticas públicas, como más tarde veremos. De este modo, el grueso de los trabajos realizados ha coincidido en destacar, de un lado, la debilidad estructural de las asociaciones de inmigrantes, y de otro, el riesgo de su dependencia y cooptación por parte de los poderes públicos. De todos modos, buena parte de estos trabajos se han centrado en las grandes asociaciones de inmigrantes asentadas en Madrid y que han estado próximas a los órganos oficiales de representación de la población inmigrante, como el Foro para la Integración Social de los Inmigrantes (a día de hoy desactivado), pero han descuidado a las medianas $y$, sobre todo, pequeñas asociaciones.

El primero de dichos trabajos, el de Veredas ${ }^{20}$, afirma que "el riesgo de cooptación de las asociaciones desde los organismos oficiales implica la supresión del debate acerca de las condiciones estructurales del ejercicio de los derechos civiles y políticos por parte de las poblaciones inmigrantes: las asociaciones quedan relegadas al ámbito de lo asistencial, maniatadas en el ejercicio reivindicativo -en caso de que tal hubiera existido- frente a quien no es sino su mentor y principal proveedor de recursos" ${ }^{21}$. En la misma línea, Martín Pérez ${ }^{22}$ destaca cómo "las asociaciones de inmigrantes desarrollan acciones que los poderes públicos delegan explícita o tácticamente en ellas, pero dependen económicamente de estos últimos y, sin embargo, apenas participan en la toma de decisiones sobre la política de inmigración y su capacidad para que las reivindicaciones que se encuentran detrás del propio hecho asociativo pasen a formar parte de la agenda política acaba dependiendo en gran medida de la posición relativa en que se han situado con respecto a los poderes públicos en las anteriores cuestiones" ${ }^{\prime 23}$. El mismo Martín Pérez reconoce que los poderes públicos han introducido a las asociaciones en lo que denomina como "un campo político de sustitución", que no constituye una verdadera vía de entrada en la política ${ }^{24}$. A conclusiones similares llegan González y Morales ${ }^{25}$ y, sobre todo, Toral ${ }^{26}$, quien en su estudio de las asociaciones de inmigrantes representadas en el Foro para la Integración Social de los Inmigrantes -órgano de carácter estatal-, concluye que "en España, la intervención del Gobierno ocupa un lugar excesivamente extenso en el ámbito

\footnotetext{
${ }_{19}$ APARICIO, Rosa, TORNOS, Andrés. Las asociaciones de inmigrantes en España. Una visión de conjunto; GARRETA, Jordi (coord.). Monográfico asociacionismo en inmigración.

${ }^{20}$ VAREDAS, op. cit.

${ }^{21}$ Ibidem, p. 222.

22 MARTÍN PÉREZ, Alberto. Las asociaciones de inmigrantes en el debate sobre las nuevas formas de participación política y de ciudadanía: reflexiones sobre algunas experiencias en España.

${ }^{23}$ Ibidem, p. 117.

${ }^{24}$ Ibidem, p. 135.

${ }^{25}$ GONZÁLEZ, MORALES, op. cit.

26 TORAL, op. cit.
} 
de las organizaciones de inmigrantes, al menos en su configuración actual. El corporativismo que la estructura de oportunidad política tiende a configurar en este país presenta numerosos peligros para la configuración de una sociedad civil sólida, ya que, sin una red horizontal fuerte y sin una participación activa de los miembros de las organizaciones, la «integración vertical» de las organizaciones puede servir para domesticar las reivindicaciones y protestas de grupos que siguen excluidos de la ciudadanía política" ${ }^{\prime 27}$. Toral incide especialmente en el papel del Estado a la hora de cooptar a las élites de las principales organizaciones de inmigrantes presentes en su territorio, mediante lo que describe como "una paradójica táctica de «integración por exclusión» en la que se integra a los líderes étnicos en instituciones carentes de poder para bloquear las posibilidades de movilización" ${ }^{\prime 28}$, al tiempo que se les resta autonomía mediante mecanismos como la gestión de las subvenciones públicas ${ }^{29}$. Una cuestión esta última, la disyuntiva de las asociaciones entre la independencia ideológica y la dependencia económica, que Masanet y Santacreu ${ }^{30}$ dicen éstas tratan de resolver adoptando una posición intermedia entre la renuncia a sus principios reivindicativos y la adopción de soluciones innovadoras para el tratamiento de la integración de los inmigrantes, precisamente a través de la participación en los foros o mesas de inmigración ${ }^{31}$.

El balance inicial que podemos hacer pues en torno al papel de las asociaciones de inmigrantes y su relación con los poderes públicos en la sociedad de acogida, al menos en la experiencia española a lo largo de los últimos años, es más bien contradictorio. Las asociaciones han tenido un papel clave en la integración social de la población inmigrante, prestando un apoyo que el Estado ha tendido a delegar en las mismas, pero ello no se ha acompañado de un reconocimiento político en paralelo, salvo para algunas grandes organizaciones que habrían experimentado dinámicas de cooptación de sus líderes, tanto en España como -y esta sería la principal novedad- en sus propios países de origen.

\section{Asociaciones de inmigrantes y sociedades de origen}

En el otro lado, muchos de los Estados de origen de los inmigrantes han desplegado en las dos últimas décadas una serie de políticas dirigidas a revincular a sus diásporas con sus sociedades de origen, tanto a nivel político, como económico, científico o cultural ${ }^{32}$. Las que se conocen como "políticas diaspóricas" también ha sido objeto de una creciente atención y resultan ser la expresión del interés

27 Ibidem, p. 126.

28 Ibidem, p. 110.

29 Ibidem, p. 112.

${ }^{30}$ MASANET, Erika, SANTACREU, Oscar. El movimiento asociativo inmigrante en la Comunidad Valenciana y sus repercusiones en la producción de capital social.

31 Ibidem, p. 168.

${ }^{32}$ WATERBURY, Myra A. Bridging the divide: Towards a comparative framework for understanding kin state and migrant-sending state diaspora politics. 
de los Estados de origen de los migrantes tanto por asegurar su lealtad, en unos casos, como por garantizar sus contribuciones económicas (remesas) o, en algunos otros, por proteger sus derechos en el exterior. La Organización Internacional para las Migraciones encargó en 2005 una encuesta dirigida a sus países miembros para conocer qué políticas mantenían en relación con sus diásporas y su participación en el desarrollo nacional. Al cuestionario, titulado "Políticas para convertir a la diáspora en agentes para el desarrollo", respondieron un total de 49 países. Como resultado, el 74\% de los países encuestados afirmaron contar con alguna estructura institucional especializada de vinculación con la diáspora (la gran mayoría de estas estructuras habían sido creadas con posterioridad al año 2000) y el 92\% declaró que disponían de políticas y programas destinados a sus propias diásporas en el extranjero. Igualmente, la investigación realizada por Gamlen, Cummings, Vaaler y Rossouw ${ }^{33}$, que analizó un total de 144 países para el período 1990-2010, detectó un considerable incremento de las instituciones nacionales vinculadas a las diásporas, cuyo número se habría aproximadamente sextuplicado. De modo que, frente al desinterés y, en muchos casos, el recelo existente en los años setenta y ochenta en torno a los migrantes por parte de los propios Estados de origen, el proceso de creación de políticas diaspóricas se habría acelerado en los últimos años en el conjunto de los países con una masa de población migrante en el exterior.

Dichas políticas han sido presentadas generalmente como un modo de promover la participación en el desarrollo nacional, de reforzar el sentimiento de pertenencia de los migrantes o, incluso, de proteger los derechos políticos y de ciudadanía de los que se encuentran en el exterior. En cambio, tampoco han faltado las críticas, al ver en las nuevas políticas una forma de fidelizar a los migrantes en el envío de remesas y, sobre todo, de extender el control del Estado fuera de las fronteras nacionales y tratar de encuadrar a la diáspora mediante programas y organizaciones afines al poder ${ }^{34}$. Esas críticas han estado especialmente presentes en países que siguen experimentando déficits democráticos internos que han podido proyectarse hacia el exterior, facilitando, por ejemplo, los envíos de dinero, pero limitando al mismo tiempo el voto de sus ciudadanos residentes en otros países.

En el caso mexicano, uno de los más estudiados, Fox y Gois ${ }^{35}$ abordan el papel de la denominada como "sociedad civil migrante" y nos advierten de los riesgos que la participación en los esquemas oficiales puede entrañar para las propias organizaciones, bien por la integración en los mismos -la concentración de proyectos en unos cuantos estados donde las organizaciones

\footnotetext{
${ }^{33}$ GAMLEN, Alan, CUMMINGS, Michael, VAALER, Paul M., ROSSOUW, Laura. Explaining the Rise of Diaspora Institutions.

${ }^{34}$ GAMLEN, Alan. Why Engage Diasporas?

${ }^{35}$ FOX, Jonathan, GOIS, William. La sociedad civil migrante. Diez tesis para el debate.
} 
de migrantes tienen una influencia política directa en los gobiernos estatales-, bien por la exclusión de aquellas organizaciones que se muestran más críticas con la agenda del desarrollo -aquellas asociaciones de inmigrantes que realizan campañas en contra de la violación de derechos humanos en México, o que están a favor de políticas de desarrollo más amplias-, bien puede verse excluidas de los planes oficiales ${ }^{36}$. Sobre esta cuestión Goldring va incluso más allá, al destacar los intentos del Estado mexicano por establecer una relación clientelar y cooptativa con su diáspora en el exterior, a través de sus políticas y programas dirigidos a las organizaciones de migrantes ${ }^{37}$. En su trabajo, Goldring discute los riesgos de clientelismo y pérdida de autonomía de las asociaciones de inmigrantes mexicanos que participan en los programas estatales, como el Tres por Uno. Literalmente habla de un partenariado neoliberal entre el Estado y las asociaciones, basado en una relación corporativista y semi-clientelar, pero que permitiría a estas últimas ganar un poder negociado ${ }^{38}$. Su conclusión es que, aunque las asociaciones no han participado directamente en el diseño del programa citado, éstas logran llevar su voz a las negociaciones y alcanzar cierta visibilidad y experiencia a través de la implementación de los proyectos, lo que conlleva una mayor capacidad de manifestar nuevas demandas al Estado ${ }^{39}$.

En general, el papel otorgado por las nuevas políticas a los migrantes en el campo del desarrollo ha levantado importantes suspicacias. A este respecto, Márquez-Covarrubias es particularmente crítico con el papel de las asociaciones de inmigrantes (clubes de oriundos para el caso mexicano) y su relación con los entes institucionales de desarrollo. Este señala cómo las prácticas de las asociaciones

se limitan a contribuir en las obras públicas y proyectos sociales en sus lugares de origen, es decir, su aporte está acotado por los programas gubernamentales, entre otros factores por el techo presupuestal anual austero, sin que hayan creado vasos comunicantes con otros sectores sociales ni hayan afianzado todavía organizaciones paralelas, por lo cual distan de ser un sujeto del desarrollo local, aun cuando puede hablarse del surgimiento de un nuevo sujeto social inscrito en la lógica del desarrollo participativo transnacional.

Y considera que las remesas colectivas y la acción de las organizaciones de migrantes "abonan al interés estatal de generar condiciones de gobernabilidad local sin proyectar institucionalmente el desarrollo" ${ }^{\prime 4}$.

Sería un debate similar al que podemos encontrar en otro país afectado por grandes flujos migratorios y un Estado que trata de extender su control, como es

36 Ibidem, p. 115.

${ }^{37}$ GOLDRING, op. cit., p. 78.

38 Ibidem, p. 94.

39 Ibidem, p. 88.

40 MÁRQUEZ-COVARRUBIAS, Humberto. Responsabilizar a los migrantes del desarrollo: lecciones del laboratorio social zacatecano. 
el caso de Marruecos, donde las asociaciones de inmigrantes han sido criticadas por asumir funciones que debería desempeñar el propio Estado (en especial la construcción de infraestructuras, como escuelas, hospitales o carreteras) ${ }^{41}$. Sin embargo, algunos autores también han puesto de manifiesto el papel de las asociaciones de inmigrantes en estos mismo países a la hora de lograr la implicación del Estado, bien en competencia común, bien en colaboración tras haber concienciado a las instituciones sobre su papel. Por ejemplo, el trabajo de Fátima Mernissi "Les Ait Debrouille"42 muestra cómo la acción inicial de asociaciones de inmigrantes y ONG locales en las montañas de Marruecos (abandonadas por el Estado durante décadas bajo el discurso de "un Marruecos inútil no desarrollable") forzó la acción de las autoridades para dar respuesta a las nuevas demandas de poblaciones concienciadas por las experiencias de mejora introducidas por las propias organizaciones. Como explica Lanly ${ }^{43}$ para el caso mexicano, las acciones de los inmigrantes pueden estimular la iniciativa local introduciendo entre la población una óptica de cambio; o, como señala Daum $^{44}$ en sus trabajos sobre el Sahel africano, las mejoras introducidas por los inmigrantes no solo son materiales, sino que también afectan a cambios en los modos de organización y participación de las poblaciones, así como en la adopción de nuevos conocimientos y competencias.

La relación de las asociaciones de inmigrantes con los Estados de origen y su trabajo allí en pro del desarrollo, son pues cuestiones que siguen resultando problemáticas, y que nos llevan a la necesidad de resituar el debate en un marco transnacional. A través de sus políticas diaspóricas los Estados tratan de regular la participación y la intervención de los migrantes en los asuntos de sus países de origen y para ello intentan modelar también el campo asociativo en el exterior, aunque parecen más interesados en promover un "nacionalismo transfronterizo" que un espíritu realmente transnacional ${ }^{45}$.

\section{Transnacionalismo y asociacionismo inmigrante: los casos de cuatro países}

En un contexto de mayor conexión entre unos y otros espacios, y de mayores facilidades técnicas para dicha conectividad, los inmigrantes y sus organizaciones han adquirido un mayor protagonismo como agentes políticos ${ }^{46} \mathrm{y}$

${ }^{41}$ LACROIX, Thomas. Les réseaux marocains du développement. Géographie du transnational et politiques du territorial.

42 MERNISSI, Fátima. Les Aït Débrouille. ONG du Haut Atlas.

${ }^{43}$ LANLY, Guillaume. Les immigrés de la vallé du fleuve Senegal en France: des nouveaux acteurs dans le développement de leur région d'origine.

${ }^{44}$ DAUM, Christoph. Les immigrés et le co-développement.

45 FITZGERALD, David. Beyond 'transnationalism': Mexican hometown politics at an American labour union.

${ }^{46}$ OSTERGAARD, Eva. Codevelopment and citizenship: the nexus between policies on local migrant incorporation and migrant transnational practices in Spain. 
de desarrollo ${ }^{47}$. Sin embargo, el papel adquirido por las asociaciones de migrantes en esos nuevos espacios también ha coincidido con un mayor interés por parte de los Estados en controlar y dar forma a los espacios transnacionales. La revisión realizada por Faist sobre el papel de los migrantes como agentes transnacionales de desarrollo señala cómo este renovado interés por convertir a los migrantes en actores diaspóricos encierra, a su juicio, problemas de fondo importantes, como la tensión entre el papel de los migrantes como agentes transnacionales de desarrollo y los intereses políticos y de control de los propios Estados de origen y destino ${ }^{48}$. A este respecto, Goldring indica que en el ámbito del transnacionalismo existe un debate entre quienes argumentan que este ofrece a los migrantes una vía para ganar autonomía y escapar al Estado nacional y quienes creen que el transnacionalismo ofrece a los Estados una oportunidad de extender y ejercer su capacidad de control cooptativo sobre las organizaciones de migrantes ${ }^{49}$.

El caso español, y el diseño e implementación en los últimos quince años de una serie de políticas conocidas con el término "codesarrollo", en conexión frecuente con los propios Estados de origen de los inmigrantes, constituye un buen ejemplo de las implicaciones de una política que ha tratado de convertir a los inmigrantes en agentes transnacionales de desarrollo. El codesarrollo, o los programas oficiales de vinculación de las migraciones con el desarrollo, han generado, en principio, una estructura de oportunidad ${ }^{50}$ para el fortalecimiento y la agencia de las asociaciones de inmigrantes. Sin embargo, el hecho de que las asociaciones hayan sido invitadas a participar en este nuevo tipo de políticas, más que a trabajar en su construcción, las ha venido situando en un papel subordinado, a la vez que un mayor nivel de representación de las asociaciones tampoco ha ido necesariamente vinculado con una mayor capacidad de agencia política ${ }^{51}$. En este sentido, Cebolla y López $^{52}$ se refieren a la persistencia de un modelo en el que las asociaciones de inmigrantes tienen reservado un papel de intermediación entre la administración y la población inmigrante, pero sin capacidad de incidir en la formulación y la implementación de las políticas públicas.

En uno de nuestros anteriores trabajos ya mostramos cómo la emergencia de un campo oficial del codesarrollo impulsado desde las instancias oficiales habría tenido como uno de sus logros una mayor visibilidad pública de las asociaciones de inmigrantes, pero al precio en buena parte de los casos de una

\footnotetext{
${ }^{47}$ FAIST, Thomas. Migrants as transnational development agents: an inquiry into the newest round of the migration-development nexus.

48 Ibidem, p. 36.

${ }^{49}$ GOLDRING, op. cit., p. 92.

50 TARROW, Sidney. El poder en movimiento. Los movimientos sociales, la acción colectiva y la política.

${ }^{51}$ GONZÁLEZ, MORALES, op. cit.

${ }^{52}$ CEBOLLA, Héctor, LÓPEZ, Ana. Transnational Latin American immigrant associations in Spain during the economic recession: a top-down model of integration and transnationalism at stake?
} 
pérdida de autonomía respecto al debate sobre la propia agenda del desarrollo y de una reorientación hacia las acciones basadas únicamente en proyectos ${ }^{53}$. Complementariamente, este hecho se acompañaría de la tendencia apuntada por Portes, cuando señala que, por un lado, las asociaciones pueden haberse visto fortalecidas por este tipo de políticas y disponer de mayores recursos para la implementación de proyectos en origen, pero, por otro lado, las mismas asociaciones se verían afectadas por comportamientos clientelares en la búsqueda de soporte gubernamental y prerrogativas financieras más allá del alcance de sus propias acciones sobre el terreno ${ }^{54}$.

Igualmente, en dicho marco las asociaciones se han visto obligadas a competir con otras organizaciones con mayor trayectoria en el ámbito del desarrollo como las Organizaciones no Gubernamentales dedicadas a la cooperación internacional. El trabajo de San Martín en España ha detectado este tipo de riesgos cuando señala que "nos encontramos el voluntarismo del trabajo asociativo frente a la estructuras profesionalizadas de las ONGD, quienes se presentan a unos proyectos que impulsa la administración y ante los que compiten en términos de desigualdad". A lo que añade que "parece evidenciarse una distancia entre la insistencia formal -en declaraciones, planes y proyectos- de la relevancia y el papel clave de los migrantes, y su reconocimiento efectivo en las actuaciones" $" 55$.

Con todo ello, y a pesar del protagonismo otorgado a las asociaciones de inmigrantes en los planes nacionales de integración (Plan Estratégico de Ciudadanía e Integración) y de cooperación para el desarrollo (Plan Director de la Cooperación Española) en relación con las nuevas líneas estratégicas de codesarrollo -en especial en los correlativos planes de nivel autonómico-, la realidad nos muestra más bien un panorama de algunos avances en un contexto de gran vulnerabilidad asociativa. En este sentido, las asociaciones de inmigrantes de Colombia, Ecuador, Marruecos y Senegal en España son un buen ejemplo de las dinámicas descritas hasta ahora. Las asociaciones de dichos países han tratado de ganar presencia pública en la sociedad de acogida a través de su incorporación a diferentes órganos de representación y participación política y social, al tiempo que han ido tejiendo vínculos con otras organizaciones y con las instancias oficiales creadas en sus países de origen.

\footnotetext{
${ }^{53}$ LACOMBA, Joan, BONI, Alejandra, CLOQUELL, Alexis, SOLEDAD, Carlos. Immigrant Associations and Co-development Policies. Among the Opportunities for Strengthening and the Risks of Cooptation in the Case of Valencia Region (Spain).

${ }^{54}$ PORTES, Alejandro, FERNÁNDEZ-KELLY, Patricia. The state and the grassroots. Immigrant transnational organization in four continents, p. 18.

${ }_{55}$ SAN MARTíN, Anna. Las asociaciones de migrantes en las actuaciones del codesarrollo. Un estudio desde la ciudad de Madrid, p. 94-95.
} 
En el caso de Marruecos, las asociaciones de inmigrantes -las más antiguas y que representan al mayor colectivo de los cuatro estudiados- nunca han logrado una representación satisfactoria en las instancias oficiales. Pese a que la mayor asociación, ATIME (hoy desaparecida por problemas en la gestión de fondos públicos), sí fue incorporada al Foro para la Integración Social de los Inmigrantes, y que otras asociaciones menores hicieron lo propio en sus respectivas comunidades autónomas (especialmente en Cataluña), ello se hizo tras un largo proceso de lucha por su reconocimiento público, en un contexto en el que la inmigración marroquí experimentó problemas de integración y un significativo rechazo social. Por su parte, sus relaciones con el país de origen tampoco estuvieron exentas de problemas, y el carácter muchas veces político de la emigración de algunos de los líderes asociativos dificultó frecuentemente la interlocución con las instancias gubernamentales en Marruecos. No obstante, el Estado marroquí experimentó tras la llegada del nuevo rey Mohamed VI un giro en las relaciones con sus nacionales en el exterior, con la incorporación del derecho al voto en la Constitución o la creación del Consejo Consultivo de los Marroquíes en el Exterior. La nueva política diaspórica del Estado marroquí ha generado nuevos espacios de participación para los emigrantes (juzgados muchas veces como insuficientes o con un papel meramente formal), y ha recibido críticas por favorecer a determinadas asociaciones y excluir a otras, o por recurrir a mecanismos de cooptación entre los líderes asociativos, incorporando algunos de ellos a los nuevos puestos creados por la administración, alimentando así la desaparición de las propias asociaciones.

Por su parte, los casos de Colombia y Ecuador guardan cierta semejanza en cuanto a su presencia en los órganos de representación política y su visibilización pública. La migración latinoamericana contó en España con una mayor aceptación social que otros flujos, y ello se ha podido reflejar en su habitual presencia en numerosos foros. Las asociaciones de inmigrantes colombianos y ecuatorianos han sido muy activas en los años previos a la crisis económica en España y han gestionado una gran cantidad de recursos públicos, tanto en proyectos de integración y formación, como en proyectos de desarrollo en los países de origen. Su dinamismo también ha sido posible, en parte, gracias al apoyo recibido desde el exterior. Las grandes asociaciones han tejido lazos con los gobiernos de origen, que han visto en ellas una correa de transmisión de sus políticas en el exterior $-y$, consecuentemente, del voto-, $y$ criticadas por ello por las pequeñas asociaciones. De hecho, algunos de sus principales líderes asociativos en España trataron de incorporarse a la vida política en los parlamentos nacionales al retornar al país de origen, u ocuparon puestos relevantes en las administraciones locales. Al mismo tiempo ambos Estados han sido muy activos -más quizás en el caso de Ecuador- en la construcción de una política de vinculación con sus diásporas y reconocimiento de su papel en el desarrollo nacional, con nuevos 
programas de inversión productiva, recuperación del talento o promoción del retorno. El resultado habría sido un relativo fortalecimiento de las asociaciones y su capacidad de agencia -proceso afectado por la crisis económica en España-, pero con un elevado coste en términos de cooptación.

En cuanto a Senegal, las asociaciones de inmigrantes en España no han alcanzado una gran envergadura o logrado un significativo reconocimiento político, pero su tejido asociativo ha sido muy dinámico y se ha caracterizado por jugar un papel clave de integración aquí (las asociaciones han desempeñado notables funciones de mediación y resolución de conflictos con la sociedad de acogida) y compromiso allí (muchas pequeñas asociaciones han puesto en marcha proyectos de asistencia y de desarrollo en sus comunidades de origen). Además de una menor dependencia de las instituciones y los fondos públicos, el carácter mayoritariamente local de las asociaciones ha permitido movilizar recursos propios y otorgar a las acciones una mayor sostenibilidad. Así, y pese a que el Estado senegalés ha sido destinatario de un importante volumen de ayuda oficial al desarrollo, y ha recibido fondos en el marco de los planes de codesarrollo europeos (en especial por parte de Francia, España e Italia), su política diaspórica ha sido más limitada que en los otros casos, y no se ha caracterizado en el mismo grado por tender al control de las asociaciones o la cooptación de sus líderes. Quizás la mayor debilidad del Estado, frente a los casos de Marruecos, Colombia o Ecuador, y la menor presencia de grandes asociaciones entre la diáspora (con muchas pequeñas asociaciones, pero conectadas por unas pocas federaciones), ha permitido establecer otro tipo de dinámica más próxima a las necesidades locales y una mayor conexión entre los migrantes y las comunidades de origen.

\section{Conclusiones}

Como hemos podido ver en los apartados anteriores las asociaciones de inmigrantes mantienen a menudo importantes vínculos tanto con los países de destino como de origen. Las asociaciones juegan un indudable papel en la integración de los inmigrantes en las sociedades de destino. De hecho, las asociaciones han asumido este papel ante la falta de mecanismos de los propios Estados, o bien estos últimos han delegado esa función y una serie de recursos para que las asociaciones se ocuparan de ello (este sería claramente el caso de la política española). Del otro lado, y si bien el mayor peso de los vínculos se establece en el espacio de las sociedades de acogida, cada vez cobran más importancia las conexiones de las asociaciones con diferentes instancias de sus lugares de salida (Estados, organizaciones de la sociedad civil y comunidades de origen), donde éstas han asumido también labores complementarias o sustitutivas a los gobiernos en programas de desarrollo. 
En este contexto se ha venido problematizando la posibilidad de dotar a las asociaciones de inmigrantes de una mayor capacidad de agencia. En este sentido, un factor clave, pero poco o nada considerado, en el debate sobre las posibilidades de fortalecimiento organizativo y capacidad de agencia de las asociaciones de inmigrantes es el de las metodologías empleadas en dichos procesos. Evidentemente, no solo los marcos políticos e institucionales en los que se plantea el apoyo a las asociaciones orientan los posibles resultados, sino también, y de forma crucial, la manera en que estos se operativizan y el modo en que los procesos son conducidos metodológicamente. Un buen ejemplo de ello es la experiencia mostrada por Escala y Rivera ${ }^{56}$ en el trabajo de formación de capacidades organizativas con asociaciones de inmigrantes latinoamericanos en Los Ángeles (California) a través de la investigación participativa. Ambos autores muestran, a partir de su trabajo de investigación e intervención con las asociaciones, cómo se puede favorecer la posición de los inmigrantes como sujetos productores de conocimiento en un proceso que facilita su participación e implicación a partir de sus propios intereses. De modo que ponen de relieve cómo la pretensión de fomentar las capacidades humanas y la agencia de los migrantes, no puede desligarse de las estrategias empleadas para ello y la concepción de la participación asociada a las mismas.

En el caso español, la ventana de oportunidad política entreabierta inicialmente por el codesarrollo ha jugado un papel fundamental en la emergencia y desarrollo organizativo de buen número de asociaciones de inmigrantes, aunque la extensión del tejido asociativo no se haya traducido necesariamente en su mayor fortaleza (la fragmentación y dispersión asociativa, así como las dificultades para encontrar una representación común, han actuado más bien en su contra). De forma sobrevenida, la crisis en España también ha tenido un impacto trascendental sobre las asociaciones de inmigrantes, con la desaparición de muchas de ellas o la reducción de su actividad a mínimos. Este panorama choca con el ímpetu previo de las asociaciones, que vieron incrementar exponencialmente su número a partir de los años 2000. Así, los planes oficiales de inmigración puestos en marcha a partir de 2004 descargaron el peso de las tareas de integración de la población inmigrante en sus propias asociaciones, financiando sus acciones y otorgando a algunas de ellas una representación política en los nuevos foros públicos creados a tal efecto. Este modelo que algunos han llamado de integración desde arriba hacia abajo o "top-down model"57 resultó especialmente útil para una serie de grandes organizaciones que adquirieron un cierto grado de profesionalización y ayudaron, voluntaria o involuntariamente, a legitimar las políticas públicas

\footnotetext{
${ }^{56}$ ESCALA, Luis, RIVERA, Gaspar. Investigación participativa, formación de capacidades y promoción del desarrollo local con asociaciones de inmigrantes en contextos transnacionales.

57 CEBOLLA, LÓPEZ, op. cit.
} 
desplegadas en los ámbitos de la integración o del codesarrollo. Estas mismas organizaciones habrían sido igualmente las menos afectadas por la crisis económica, pese a su notable dependencia económica de fondos públicos y el recorte de los mismos, al disponer de mayores contactos con las administraciones y, sobre todo, haber podido reorientar su acción hacia nuevos ámbitos como el retorno de los inmigrantes, objeto de las nuevas políticas ${ }^{58}$. Pero también las asociaciones más pequeñas, con una menor dependencia de fondos públicos y conectadas con vínculos comunitarios de mayor calado con sus miembros, habrían mostrado una importante capacidad de resistencia y resiliencia frente a la crisis, como muestra particularmente el caso de Senegal. Por el contrario, las asociaciones de tamaño medio habrían sido las principales damnificadas, al conjugar su dependencia económica de los fondos públicos con un escaso peso en los foros de representación.

Lo que debería haber sido en el caso español una oportunidad para el fortalecimiento de la capacidad de agencia transnacional de las asociaciones de inmigrantes, se ha visto en buena medida frustrada por dos razones de fondo, si dejamos de lado el efecto coyuntural de la crisis: la primera, la propia agenda del Estado de recepción y los respectivos Estados de origen de los migrantes, dirigida más a orientar la acción de los migrantes que a fortalecerla; y la segunda, la incapacidad de las asociaciones de inmigrantes de articular una voz común, ejercida ésta muchas veces por las grandes asociaciones sin un consenso previo.

\section{Bibliografía}

APARICIO, Rosa; TORNOS, Andrés. Las asociaciones de inmigrantes en España. Una visión de conjunto. Madrid: Ministerio de Trabajo e Inmigración, 2010.

BRETÓN, Víctor. Cooperación al desarrollo y demandas étnicas en los andes ecuatorianos. Quito: FLACSO, 2001.

CASTLES, Stephen; MILLER, Mark J. La era de la migración Movimientos internacionales de población en el mundo moderno. México: Miguel Ángel Porrua, 2004.

CEBOLLA, Héctor; LÓPEZ, Ana. Transnational Latin American immigrant associations in Spain during the economic recession: a top-down model of integration and transnationalism at stake? In AYSA LASTRA, María; CACHON, Lorenzo (eds.). Immigrant vulnerability and resilience. Switzerland: Springer International Publishing, 2015.

DAUM, Christoph. Les immigrés et le co-développement. CERAS Projet, n. 288, 2005.

ESCALA, Luis; RIVERA, Gaspar. Investigación participativa, formación de capacidades y promoción del desarrollo local con asociaciones de inmigrantes en contextos transnacionales. Cuadernos Electrónicos del Desarrollo Humano y la Cooperación, n. 6, 2016 (en prensa).

${ }^{58}$ Ibidem, p. 178. 
FAIST, Thomas. Migrants as transnational development agents: an inquiry into the newest round of the migration-development nexus. Population, Space and Place, v. 14, n. 1, 2008, p. 21-42.

FITZGERALD, David. Beyond 'transnationalism': Mexican hometown politics at an American labour union. Ethnic and Racial Studies, v. 27, n. 2, 2004, p. 228-247.

FOX, Jonathan; GOIS, William. La sociedad civil migrante. Diez tesis para el debate. Migración y Desarrollo, v. 7, n. 15, 2010, p. 81-128.

GAMLEN, Alan. Why Engage Diasporas? ESRC Centre on Migration, Policy and Society, Working Paper, n. 63, 2008.

GAMLEN, Alan; CUMMINGS, Michael; VAALER, Paul M.; ROSSOUW, Laura. Explaining the Rise of Diaspora Institutions. IMI Working Papers Series, n. 78, 2013, p. 1-33.

GARRETA, Jordi (coord.). Monográfico asociacionismo en inmigración. Revista Internacional de Sociología, Vol. 71, no extra 1, 2013, p. 4-214.

GOLDRING, Luin. The mexican state and transmigrant organizations: Negotiating the boundaries of membership and participation. Latin American Research Review, v. 37, n. 3, 2002, p. 55-99.

GONZÁLEZ, Amparo; MORALES, Laura. Las asociaciones de inmigrantes en Madrid. Una nota de investigación sobre su grado de integración política. Revista Española del Tercer Sector, n. 4, 2006, p. 129-173.

HIRSCHMAN, Albert O. Salida, voz y lealtad. Respuestas al deterioro de empresas, organizaciones y estados. México: Fondo de Cultura Económica, 1997.

ISKANDER, Natasha. Creative State: Forty Years of Migration and Development Policy in Morocco and Mexico. Ithaca: Cornell University Press, 2010.

LACOMBA, Joan; BONI, Alejandra; CLOQUELL, Alexis; SOLEDAD, Carlos. Immigrant Associations and Co-development Policies. Among the Opportunities for Strengthening and the Risks of Cooptation in the Case of Valencia Region (Spain). Voluntas. International Journal of Voluntary and Nonprofit Organizations, v. 25, n. 4, 2014.

LACROIX, Thomas. Les réseaux marocains du développement. Géographie du transnational et politiques du territorial. Paris: Les Presses de Sciences Po, 2005.

LANLY, Guillaume. Les immigrés de la vallé du fleuve Senegal en France: des nouveaux acteurs dans le développement de leur région d'origine. Land Reform, n. 1, 1998, p. 106-120.

LUSSI, Carmem; MARINUCCI, Roberto. O protagonismo dos brasileiros no exterior e seu papel na defesa e promoção dos próprios direitos. Resultados de uma pesquisa junto a organizações e lideranças de brasileiros e brasileiras no exterior. REMHU, Revista Interdisciplinar de Mobilidade Humana, n. 31, 2008, p. 119-142.

MÁRQUEZ-COVARRUBIAS, Humberto. Responsabilizar a los migrantes del desarrollo: lecciones del laboratorio social zacatecano. Economía, Sociedad y Territorio, v. 10, n. 32, 2010, p. 99-141.

MARTÍN PÉREZ, Alberto. Las asociaciones de inmigrantes en el debate sobre las nuevas formas de participación política y de ciudadanía: reflexiones sobre algunas experiencias en España. Migraciones, n. 15, 2004, p. 113-143. 
MASANET, Erika; SANTACREU, Oscar. El movimiento asociativo inmigrante en la Comunidad Valenciana y sus repercusiones en la producción de capital social. Migraciones, n. 27, 2010, p. 49-81.

MERNISSI, Fátima. Les Aït Débrouille. ONG du Haut Atlas. Casablanca: Le Fennec, 1997. MOYA, José C. Immigrants and associations: a global and historical perspective. Ethnic and Racial Studies, v. 31, n. 5, 2005, p. 833-864.

OSTERGAARD, Eva. Codevelopment and citizenship: the nexus between policies on local migrant incorporation and migrant transnational practices in Spain. Ethnic and Racial Studies, v. 34, n. 1, 2011, p. 20-39.

PORTES, Alejandro; FERNÁNDEZ-KELLY, Patricia. The state and the grassroots. Immigrant transnational organization in four continents. New York-Oxford: Berghahn, 2015.

REX, John; JOLY, Danielle; WILPERT, Czarina. Immigrant Associations in Europe. Aldershot: Gower, 1987.

SAN MARTÍN, Anna. Las asociaciones de migrantes en las actuaciones del codesarrollo. Un estudio desde la ciudad de Madrid. Migraciones, n. 30, 2011, p. 71-99.

SASSEN-KOOB, Saskia. Formal and informal associations: Dominicans and Colombians in New York. Center for Migration Studies (special issues), n. 7, 1989, p. 261-277.

SEN, Amartya. Desarrollo y libertad. Barcelona: Planeta, 2000.

TARROW, Sidney. El poder en movimiento. Los movimientos sociales, la acción colectiva y la política. Madrid: Alianza Universidad, 1997.

TORAL, Guillermo. Las asociaciones de inmigrantes como sociedad civil: un análisis tridimensional. Revista Española de Investigaciones Sociológicas, n. 132, 2010, p. 105-130.

VEREDAS, Sonia. Las asociaciones de inmigrantes en España. Práctica clientelar y cooptación política. Revista Internacional de Sociología, n. 36, 2003, p. 207-225.

WATERBURY, Myra A. Bridging the divide: Towards a comparative framework for understanding kin state and migrant-sending state diaspora politics. In BAUBÖCK, Rainer; FAIST, Thomas (eds.). Diaspora and Transnationalism. Concepts, Theories and Methods. Amsterdam: Amsterdam University Press, 2010.

Recibido para publicación en 25.06.2016

Aceptado para publicación en 10.08.2016

Received for publication in June $25^{\text {th }}, 2016$

Accepted for publication in August 10 $0^{\text {th }}, 2016$

ISSN impresso 1980-8585

ISSN eletrônico 2237-9843

http://dx.doi.org/10.1590/1980-85852503880004703 